\title{
LUGAR PREFERENTE DE LA NOVELA CORTA EN LA LITERATURA NORTEAMERICANA
}

\author{
María Antonia Álvarez Calleja
}

UNED

Aunque ya desde que el ser humano comenzara a comunicarse siempre haya existido el relato breve, oral o escrito -cuento, parábola, fábula- y a finales del XVIII contáramos con tantos relatos góticos y de otros temas, el lugar preferente que ha conseguido la novela corta en la literatura norteamericana tiene una historia reciente: hasta la publicación en 1819 de The Sketch Book, de Washington Irving, no desempeñó este papel trascendental. Irving consiguió crear unos personajes que son legendarios en las letras americanas: entre ellos el perezoso, holgazán, indolente, pero simpático y servicial «eterno adolescente», que se evade de la realidad a través del sueño. Como afirma Philip Young, Rip se ha convertido en «the symbol for the sleep that has been his life ${ }^{l}$ » $y$, por esta razón, Irving merece ser el patriarca del género. No obstante, esta paternidad tiene que compartirla con varios escritores, entre otros, Nathaniel Hawthorne, E. A. Poe, o Fiztgerald, el creador de esos inovidables personajes que encarnan el «sueño americano».

La novela corta es una forma de presentar de manera inteligible los diversos aspectos americanos, de mostrar la diversidad de la vida de su pueblo, y es-

1 Young, Philup. 1976 «Fallen From Time: Rip Van Winkle», en Myers, Andrews B. (ed.) 1860-1974 A century of Commentary of the Works of Washington Irving. Tarrytown, NY: Sleepy Hollow Restorations, pág. 477. 
to le ha convertido en una expresión artística natural y espontánea. No hay que negar la curiosa paradoja de que Norteamérica, una nación grande y diversa, tenga esta preferencia por la novela corta y se exprese mucho mejor a través de «a particular scene an individual personality revealed by a short story than in the prawling abundance of landscape and the crowded scenes presented in a novel $^{2}$ \%.

Es en esta preocupación por lo particular donde la novela corta norteamericana adquiere universalidad -cualquiera que sea su estilo, perspectiva o propósito-, ya que los relatos más famosos del pasado y del presente acercan al lector a su tierra haciéndole partícipe de sus experiencias básicas. Y de ahí que la aparición de la novela corta como forma literaria en la América del siglo XIX no resulte sorprendente, pues, como ha hecho notar Frank O'Connor:

The short story flourishes in «marginal» cultures and areas, and it deals very often with submerged population groups and with figures who find themselves situated within «frontier» or «outsider» conditions, and there is also «local colour» writing ${ }^{3}$.

$\mathrm{Y}$ todas estas características son las que le han proporcionado el marco adecuado para poder mostrar su variedad e innovación sorprendentes y crear unos personajes inolvidables.

\section{El TeMa del SueÑo como Evasión de la REALIDAD}

Es muy común en el mito, la leyenda y el folklore el tema de una persona que permanece durmiendo durante un largo período de tiempo y, cuando despierta, descubre que han cambiado todas las circunstancias anteriores y han ocurrido acontecimientos que desconoce. Este tema del sueño ha pasado de una tradición literaria a otra a través de la historia, estando presente en la mayoría

2 Sullivan, Nancy. 1981. Introducción a The Treasury of American Short Stories. Garcen City, New York: Doubleday and Company, Inc., pág. XVII.

3 O'CONNOR, Fl.ANNERY. 1963 The Lovely Voice: A Study of the Short Story. London: Macmillan. 
de los cuentos sobrenaturales. Tantos héroes inovidables - Arturo, Merlín, Carlomagno, Guillermo Tell, el dios nórdico Odin, el pastor Endymion- y otras tantas heroínas míticas - la bella durmiente del bosque, Brunilda-, han convertido el tema del sueño en un elemento mítico de la fícción, adquiriendo incluso la cualidad de algo real.

El mito del sueño se ha extendido por todo tipo de literaturas, hallándose presente en libros tan diferentes como el Corán, la Biblia o una novela de Mark Twain: Innocent Abroad. Este tema del sueño ha aparecido ya en tantos relatos, que el lector se ha acostumbrado a admitirlo como un elemento necesario para que la ficción pueda producir toda clase de extraños acontecimientos.

Aunque el prototipo de todos los héroes de estos mitos y leyendas sobre durmientes sea Odín $\rightarrow$ Woden-, dios de la muerte. «Rip Van Rinkle» ${ }^{4}$, de Washington Irving es el más conocido. Entre sus antecedentes están los siete durmientes de Éfeso, antiguos cristianos perseguidos por el Emperador Decius que se escondieron en una montaña y se quedaron dormidos; al despertar, creyeron que sólo había transcurrido una noche y uno de ellos fue hasta la ciudad para comprar pan, pero, cuando llegó allí, quedó sorprendido al divisar una cruz sobre la puerta de entrada oír hablar libremente de Jesús. La sorpresa mayor la recibió al pagar el pan con las monedas que tenía en el bolsillo, pues comprobó que no estaban en curso legal desde hacía 360 años. La historia más próxima de «Rip» es la aventura de Herla, rey de los bretones, y su fuente directa, según Andrews B. Myers ${ }^{5}$, es un mito sobre el anciano griego llamado Epimenides, que en 1496 desarrollo Erasmo.

No obstante, Washington Irving utiliza el tema del sueño de una forma muy diferente a sus antecesores ya que, por medio del sueño, el protagonista puede lograr su más ferviente deseo: al permanecer dormido durante veinte años, Rip evita hacerse adulto, lo que le obligaría a realizar todas las tareas que odia como son cuidar la granja, educar a sus hijos y obedecer a su mujer. Según afirma Philip Young:

It is not just his wife he has dodged, either, but all the obligations of maturity: occupation, domestic and financial responsibility, a political position, duty to society in a time of war ${ }^{6}$.

Winkle.

${ }^{4}$ IRVING, WASHINGTON. 1819. «Rip van Minkle», The Sketch Book. New York: C.S. Van

5 MYERS, ANDREw B. (1976) A Century of Commentary on the Works of Washington Irving. Tarrytown, New York: Sllepy Hollow Restorations, Inc.

6 Young, Philip, ob. cit., pág. 477. 
El héroe creado por Washington Irving simboliza al primer padre americano que prefiere continuar toda su vida siendo el hijo en vez del progenitor: «it is clear that Rip escaped no change of life, but his very manhood - went from chilhood to second childhood with next to nothing in between» ${ }^{7}$. Washington Irving lo explica claramente al final de la historia:

Having nothing to do at home, and being arrived at that happy age when a man can do nothing with impunity, he took his place once more on the bench, at the inn door, and was reverenced as one of the patriarchs of the village, and a crhonicle of the old times ${ }^{8}$.

En realidad, su relación con los acontecimientos que se desarrollan en la historia es tan ambigua, que cuando vuelve al pueblo «charming and infantile», Irving le dejará que siga siempre haciendo lo que más le gusta: «he will tell his tale of twenty years sleep at Mr. Do-Littel's ${ }^{9}$.

En «Young Goodman Brown ${ }^{10}$ ", de Nathaniel Hawthorne, el tema del sueño no produce resultados positivos como en la obra anterior, sino un cambio de actitud en Goodman Brown que lo convierte en un extraño entre sus amigos y familiares.

El protagonista de Hawthorne se presenta al principio de la historia como un muchacho simple y de buen carácter que acude al bosque para tratar de enfrentarse al diablo y recuperar su libertad. No obstante, a causa de la aparición de su amada esposa Fe en el aquelarre, cambia su carácter y se convierte en el principal horror de la escena:

«It was a dream of evil omen for young Goodman Brown. A stern, a sad, a darkly meditative, ad distrustful, if not a desperate man did he become, from the night of that fearful dream.»"l

7 ibid.

8 IRVing, Washington. 1819. «Rip van Winkle», ed. cit. pág. 512.

${ }^{9}$ Ibid.

10 Hawthorne, Nathaniel. 1937 (1835) «Young goodman Brown" The Complete Novels and Selected tales of Nathaniel Hawthorne. New York: The Modern Library.

11 Ibid, pág. 501. 
A pesar de los resultados contradictorios, en ambas historias el sueño colma las ambiciones de los dos protagonistas: para Rip siempre ha sido su sueño dorado escapar de la madurez y de todas las ataduras que le ha acarreado su matrimonio, y a Goodman el sueño le permite aparecer como el único individuo del pueblo que ha rehusado pecar, lo que confirma sus buenas cualidades y su bondad, frente a los pecadores que le rodean.

\section{LiBERTAD vS AMOR EN LA LITERATURA FEMENINA}

Hasta el siglo XX, las mujeres escritoras se vieron relegadas a una clase aparte, donde exclusivamente se las comparaba con otras de su mismo sexo y, como afirma Josephine Donovan, «were, however, put down by the male authors» 12 .

Incluso Nathaniel Hawthorne, celoso quizás de su éxito comercial, se refería a ellas como «dammed mob of scribbling women» ${ }^{13}$.

Desde 1820 a 1870, según Nina Byam, «women's fiction was an identifiable genre, one that focused on the trials and tribulations of a noble heroine winning her own way in the world» ${ }^{14}$. En realidad, las mujeres escribieron de forma prodigiosa sobre el tema sentimental, debiendo ajustarse a unas normas establecidas: el ideal femenino era una mujer delicada, que sabía entretener de una manera inteligente y mostrar su modestia tanto en la forma de hablar como en los temas elegidos.

Para la mujer del siglo XIX, llegar a la edad adulta suponía la pérdida del yo $\mathrm{y}$, al contrario que los hombres - que lograban la plenitud en la madurez-, la mujer añoraba su época infantil, recordando qué libre se sentía a esa edad y cómo había perdido después su libertad para depender del marido. Durante sus primeros años, todo había sido satisfacción personal, todo era posible: incluso se les animaba a desarrollar sus deseos de independencia, que la realidad se encargaba de truncar: «for the women the only choice, the sacrifice required for survival, is to give up a woman's proper life and cover the default of the men,

12 Donovan, Josephine. 1980. Sarah Orne Jewett. New York: Frederick Ungar Publising Co., pág. 1.

13 Ibid.

14 BAYM, NinA. 1978. Women's Fiction: A Guide to Novels by and about Women in America, 1820-1870, Ithaca, New York: Comell University Press, pág. 11. 
to be guardians and preservers of a community with no other source of vitality and support» ${ }^{15}$. No obstante, añade Heather Mc Clave:

... with the rise of New Criticism, deeply allied to modernist ideals of the impersonality of the artist and the intrinsic significance of myth and art... at least in theory, women gain the option of being judged as artists first, and over all they seem to grow in confidence and stature in this professional role ${ }^{16}$.

Podemos analizar este tema «libertad vs amor» a través de los cuentos de dos escritoras del siglo XIX, Sarah Orne Jewett y Kate Chopin. En «A White Heron» ${ }^{17}$-que para Gwen L. Nagel es "perfect in its way, a tiny classic»" ${ }^{18}$-, Sarah Orne Jewett expone el deseo de la heroína de interrumpir su desarrollo hacia la edad adulta. Nos dice cómo Sylvia, una niña solitaria que no ha tenido amigos, excepto los animales y las plantas, se encuentra ante un arrogante coleccionista de pajaros $y$ «the girl's lonely heart goes out to this gracious romanthic stranger» ${ }^{19}$. Por primera vez esta niña siente los caprichos del amor, pero no se rinde a él porque prefiere continuar siendo, para siempre, la virgen libre del bosque, fiel a sus principios de libertad, y leal a todos los animales salvajes que allí viven:

She remenbers how the white heron came flying through the golden air and how they watched the sea and the morning together, and Sylvia cannot speak; she cannot tell the heron's secret an give its life away ${ }^{20}$.

15 McClave, Heather (ed.) 1980 Women Writers of the Short Story. Englewood Cliffs, N. J.: Prentice-Hall, Inc., pág. 18.

16 Ibid, pág. 9.

17 JeWETt, SARAh ORNe 1988 (1886) «A White Heron», A White Heron and Other Stories (The Collected Works of Sarah Jewett) US: Houghton.

18 NAGEL, GWEN. 1984 Critical Essays on Sarah Orne Jewett. Boston, Mass.: G. K. Hall \& Co., pág. 34.

19 Mathiessen, FrRancis Otto. 1929 Sarah Orne Jewett. Boston \& New York: Houghton Miffling Co., pags. 82-3.

20 JeWett, SARah ORNe. 1988 (1886) *A White Heron», pág. 595. 
En «The Story of an Hour» ${ }^{21}$ Kate Chopin va más allá de las convenciones de la ficción y la sociedad de su época, y su análisis de la forma en que las mujeres se vean a sí mismas, o permiten que los demás las vean, es sutil y lleno de implicaciones. Trata de la complejidad de un fuerte deseo de independencia, comparable a un renacer. Según Judie Newman:

Her touch is light, umprescriptive and often humorous. The «technique» fo her story serve - subvertingly and with striking artfulness - to render the whole complex problematic of fitting self to image. While she is not polemicist, her story suggest that women are amply capable of enlarging the roles available to them, and that active accomodation trascends empty conformity or withdrawal into one or another pre-ordained fan$\operatorname{tasy} 22$.

Retrata una esposa que muere de dolor cuando descubre que a su marido no lo han matado en un accidente, y ella no podrá volver a sentir la libertad que ha disfrutado durante «una hora». Per Seyersted considera esta obra «the most startling picture of female self-assertion ${ }^{23}$, donde la protagonista rehusa su vida oscura en la que jamás podrá llegar a realizarse. La señora Mallar ha descubierto que ningún tipo de amor o seguridad puede compensarle la falta de control de su propia existencia:

«Free! Body and soul free!» There would be no one to live for her during those coming years; she would live for herself ${ }^{24}$.

El tema del amor, pues, en la primera historia empieza con la iniciación y termina con la renunciación, y en la segunda hay desilusión y un gran deseo de independencia. El estilo es subjetivo en la primera e impersonal en la última. El tono del narrador es benévolo y crítico en la primera, e irónico en la segunda,

21 ChOPIN, KATE. 1969 (1890) «The Story of an Hour, Complete works of Kate Chopin. US: Southern Library Studies.

22 Newman, Judie. 1985 «Kate Chopin: Short Fiction and the Arts of Subversion», en A. Robert Lee (ed.) The Nineteenth-Century of American Story, ob. cit., pág. 162.

23 Seyersted, Per. 1969 Kate Chopin: A Critical Biography. Baton Rouge, pág. 111.

24 CHOPIN, KATE. 1969 (1890) «The Story of an Hour», ed., cit., pág. 179. 
pero ambas tratan del tema de la libertad, porque las dos han sido escritas por mujeres y éste era su principal problema en el siglo XIX.

Por ese motivo, los personajes masculinos de ambas historias se presentan de forma impersonal: en «Story of an Houn» conocemos el nombre del esposo, Brentily Mallar, aunque no sepamos casi nada más de él; solo que entró «a little travel-stained, composedly carrying his gripsack and umbrella»; esta única descripción es el retrato acabado de un caballero frío, rígido y perfectamente correcto, que no despierta la pasión de su joven y bella esposa. Y en «A White Heron» el joven no merece un nombre, es un tipo, y los calificativos que se le dedican cambian de acuerdo con la actitud de la autora: al principio es el «enemigo», porque recuerda al "great re-faced boy who used to chase and frighten her" y lleva una escopeta; más tarde se convierte en un amigo, y al final es el ideal de la joven o el principal objeto de su admiración. Ante el cazador «the woman's heart, asleep in the child, was vaguely thrilled by a dream of love» ${ }^{25}$, y se mueve con una premonición del poder y la liberación del amor, pero, según Mc Clave:

... in defence of her mysterious sympathy with wild creatures and her secret knowledge of the heron's sanctuary, she refuses his appeal like a profaning courtship (in his offering her money is again the imputation of grossness) and the suggestion is made that her whole life will turn of this renunciation ${ }^{26}$.

En la comunión con la garza blanca ha entrado en posesión de una lealtad mucho mayor que no venderá al mundo. Sylvia reafirma su comunión anterior con la naturaleza, pero - como afirma Peggy Skaggs- lo hace consciente de su elección y de lo que pierde: «Loyalty to the young man would bring companionship but also submission to another's lead, a taming of Sylvia's spirit and an acceptance of values not her own ${ }^{27}$. Su lealtad a esos valores la separa del "gran mundo" pero en compensación la otorga su comunión con el mundo natural. En la sociedad sin futuro para la mujer del siglo XIX, el instinto femenino sólo puede llenarlo la devoción a lo permanente: sus energías sólo pueden destinarse a conservar el pasado, a colaborar en la preservación de la naturaleza. Este es el mensaje de $A$ White Heron, donde la heroína es sólo una niña, pero que ha recibido su iniciación a través de la aventura vivida.

25 JewetT, SaRah ORNe. 1988 (1886) «A White Heron», ed., cit., pág. 593.

26 McClave, Heather (ed.) 1980 Women Writers of the Short Story, ob. cit., pág. 18.

27 Skaggs, Peggy. 1985 Kate Chopin. Bston: Twayne Publishers, pág. 160. 


\section{ACtualización del TeMa del Terror de la Novela Gótica}

La novela corta resulta una forma muy apropiada para los argumentos de terror, ya que el autor sólo tiene que abocetar una situación trágica sin explicar los detalles, que harían la historia excesivamente morbosa. En los dos relatos siguientes, todas las palabras responden, directa $o$ indirectamente, a un diseño preestablecido de presentar una historia terrorífica: ambas crean una atmósfera misteriosa en la que los personajes están totalmente desconectados del mundo exterior, y en ambas hay crímenes, misterio, locura, alucinación. Muerte.

"A Rose for Emily» 28, afirma Hans H. Skei, «is a gothic tale in the sense that it creates a doom-laden atmosphere where an old spinter who is totally shut out from the teeming outside world lives in a decaying mansion with one of the floors closed.» ${ }^{29}$ Aquí William Faulkner nos presenta un conflicto entre una joven y la inflexible autoridad paterna. El tema principal es —como en todas sus obras- el declinar del pasado romántico del Sur; pero además de las connotaciones psicológicas que lleva asociadas, se trata de un cuento terrorífico imaginario, que tiene lugar en la mansión elegante y lujosa de una antigua familia sureña. En el argumento están entrelazados el amor, la muerte y la locura con la «rosa» como símbolo de pasión, aunque aquí sólo produzca un terror tan estremecedor que probablemente no iguales ninguna de sus obras.

Esta novela corta es de gran importancia en la obra de Faulkner, pues, según Skei:

It was the author's first tale in his major period as a short story writer, and it is probably to this very fact that much of the responsibility of the author's more frequent and better organized attempts to market his short stories can be attributed ${ }^{30}$.

Los principales elementos están combinados de tal manera, que consiguen dar unidad al tema: durante muchas décadas, Emily había sido la última

28 Faulkner, William. 1967 (1950) «A Rose for Emily», The Portable Faulkner, Malcolm Cowley (ed.) US: The Viking Press.

29 Skel, Hans H. 1985 William Faulkner: The Novelist as Short Storu Writer Oslo: Universitestforlaget. Publications of the American Institute, pág. 12.

301984 (1981) William Faulkner: The Short Story Career. Oslo: Universitestforlaget. Publications of the American Institute, pág. 51. 
y única representante de los nombres augustos de la ciudad; había constituido una tradición, un deber y una carga; una especie de obligación hereditaria sobre los habitantes ordinarios, que le habían considerado un idolo y la habían eximido de pagar los impuestos a los que todos los ciudadanos estaban obligados.

Sin embargo, lo que el pueblo no podía aceptar era que una Grierson se enamorara de un yanqui. Siempre la había apartado su padre de todos los pretendientes y de ahí pueden derivarse sus problemas con los hombres: la «rosa para Emily", según anuncia el título de la historia, estaba en la habitación que nadie había visto durante cuarenta años:

Upon this room decked and furnished as for a bridal; upon the valance curtains of faded rose color, upon the rose-shaded light ... upon a chair hung a suit, carefully folded; beneath it the two mute shoes and the discarded socks... The man himself lay in the bed. ${ }^{31}$

Este es el horror de la historia, que miss Emily -con su poder y con el respeto e intimidad impuestos sobre los habitantes del pueblo- ha podido ocultar un crimen horrible y conservar al joven para siempre, no descubriéndolo el lector hasta el final: «the body had apparently once lain in the attitude of an embrace» 32 .

No obstante, el tema principal de la historia, el yanqui derrotado por el hombre del Sur, sólo se completa con las líneas finales: «... but now the long sleep that outlasts love, that conquers even the grimace of love had cuckolded him» ${ }^{33}$ : es decir, Emily no había estado durante años adorando a un hombre del Norte en su habitación «rosa», sino que «el largo sueño» -el somnoliento Sur- era quien había ocupado su lugar.

En la segunda historia "The Tale-Tell Heart» ${ }^{34}$, uno de los mejores relatos cortos de Poe, el terror es psicológico y tan complicado como en todas sus novelas, ya que crea una estructura de perspectivas alrededor del crimen. Desde el principio de la historia hay implicaciones entre el autor y el narrador, y también entre el narrador y el lector. El comienzo es sorprendente:

31 Faulkner, William. 1967 (1958) «A Rose for Emily», ed. cit., pág. 226.

32 Ibid, pág. 226.

33 Ibid.

34 Poe, Edgar Allan. 1946 (1843) «The Tell-Tale Heart» The Complete Poems and Storic's fon E. A. Poe. New York: A. A. Knopf. 
«True! - nervous- very, very dreadfully nervous I had been and am! but why will you say that I am mad? The disease had sharpened my senses - not destroyed- not dulled them. ${ }^{35}$

Es decir, incluso antes de que el lector piense que el protagonista y narrador está loco, él lo niega. El vocabulario ha incorporado palabras góticas como: diabólico, terror, noche, lúgubre, y la atmósfera es sofocante, ya que leemos con frenesí las descripciones fantásticas de su espera obsesionada en la oscuridad, hasta que la víctima queda paralizada. No podía matar al viejo cuando tenía el ojo cerrado, necesitaba ver el ojo diabólico; pero lo que no puede soportar es el creciente sonido del corazón: «... the sound increased - and what could I do? It was a low, dull, quick sound- much such a sound as a watch makes when developed in cotton» ${ }^{36}$.

La estructura de los dos cuentos y sus temas pueden ser diferentes - según Skein: «show a growing sophistication; a growing awareness of economy in the presentation and hadling of a short story, and improved craftsmanship» ${ }^{37}-$, pero ambas son historias de terror y fueron escritas justamente para eso, para llenar al lector de horror con sus finales terroríficos. Cuando todos los diversos elementos de estos relatos cortos de Faulkner y Poe desaparecen, ese horror permanecerá en la mente del lector.

\section{LOS «LOCOS» AÑOS VEINTE Y EL «SUEÑO AMERICANO»}

F. Scott Fiztgerald, según Sheilah Grahamm «is the writer of the gay twenties, of family youth, of bobbed hair and short skirts and crazy drinking-the hazz age» ${ }^{38}$ y el hecho de que se le llamara «the Laureate of the Jazz Age» demuestra que era una figura popular. No obstante, por esta popularidad tuvo que pagar un alto precio, como muestra el olvido de la crítica: «the surface glamour of his work oscured its real significance, until recently» ${ }^{39}$. En realidad, la aten-

35 Ibid, pág. 13.

36 Ibid, pág. 17.

37 SKEI, HNAS H. 1984 (1981), op. cit., pág. 51.

38 Graham, Shellah \& Frank, Gerold. 1959 Beloved Infidel. London, pág. 140.

39 Sivaramkrishna, M. 1978 Icarus of the Jazz age. H-y-derabad: Osmania University, pág. 1 . 
ción prestada por la crítica a sus novelas cortas es abundantísima, pero son breves notas sin comentarios profundos. El número de ensayos críticos serios, según Jackson Bryer ${ }^{40}$, probablemente no pase de veinte.

En la novela corta «Bernice Bobs her Hain ${ }^{39}$, el comportamiento de los adolescentes de Fiztgerald anuncia el de los adultos de The Great Gatsby (1924). Su actitud es similar, sólo piensan en bailar, divertirse y disfrutar del momento actual, antes de que sea demasiado tarde:

... they show the possibilities of excitement and pleasure dulled by a narrowness of outlook and an elaborate social ritual only half-concealing a lack of inner substance ${ }^{42}$.

Para triunfar entre estos jóvenes, no hay que hablar de cosas serias, pues se desprecia a los que sean ajenos a esas reglas o sigan las normas de la vieja generación. Esta juventud inquieta está convencida de que todos los dioses han muerto y no habrá otra guerra mundial; pero bajo la superficie de esta «jazz generation» sólo hay tristeza, vacío, frustración, rivalidad y soledad:

No matter how beautiful or brilliant a girl may be, the reputation of not being frecuently cut in on makes her position at a dance unfortunate ... youth in this jazz-nourished generation is temperamentally restless ${ }^{43}$.

Aunque el tema principal del relato, al igual que en la novela, sea el «sueño americano». Fiztgerald hace una crítica de la riqueza, de los privilegios, de los excesos. Desde el principio, el escritor utiliza varias estrategias técnicas para establecer el tono, la forma y el tema de la historia. El modo de hablar de todos los personajes es exagerado hasta el ridículo, usando palabras poco adecuadas para unos personajes que ya hace muchos años pasaron su niñez: «terrifying

40 Bryer, JaCkson R. (ed.) 1982 The Short Stories of F. Scott Fitzgerald: New Approaches in Criticism. Madison, Wisconsin: The University of Wisconsin Press, Ltd., pág. XI.

${ }^{41}$ Fizgerald, F. SCOTT. 1920 «Bernice Bobs Her Hair», Flappers and Philosophers. US: Charles Scribner's Sons.

42 WaY, Brian. 1980 F. Scott Fitzgerald and the art of Social Fiction. London: Edward Arnold (Publishers) Ltd., pág. VII.

43 Fuzgerald, F. SCOTt. 1920 «Bemice Bobs Her Haim», ed. cit., pág. 569. 
fox trots», «l'm obliged loads» «... collapsed and howled with glee». Los tres protagonistas que forman el triángulo de la historia no están estudiados psicológicamente como individuos, al contrario de la mejor novela de Fiztgerald: son estereotipos que encarnan tres clases diferentes de adolescentes en los «locos» años veinte.

Las jóvenes heroínas de Fiztgerald, según Brian Way «assert their independent wills and exploit their sexual attractiveness with complete impunity: indeed their social success is in exact proportion to the daring and high-handedness they display in doing as they please $"{ }^{44}$. Marjorie es un personaje vacío con pocas cosas que ofrecer en una relación humana, un ser irresponsable que sacrifica todo lo que la rodea a fin de poder continuar sus éxitos: de todas formas, no es tan claramente corrupta como Daisy, en The Great Gatsby. Y Bernice, como Gatsby, encarna el tipo de joven provinciana que quiere convertirse en un miembro popular de la sociedad que la rechaza. Si Gatsby es el arribista social que trata de hacer realidad el «sueño americano", Bernice sólo desea ser aceptada como un miembro del grupo, al igual que lo ha venido siendo en su propio entorno social.

Warren es el elemento masculino, que desarrolla uno de los temas principales de la historia: la ambigüedad del amor. Si Warren ama a Marjorie, es porque la joven simboliza todos sus sueños, y de ahí que se produzca un cambio inmediato de pareja cuando Bernice pasa a ocupar su lugar.

Otro tema importante de la historia es la soledad de estos jóvenes que siempre están en grupo. Fiztgerald capta lo artificial de una sociedad que ha olvidado la gratitud. En The Great Gatsby cuando muere el héroe, no hay personas que le lloren en el entierro, todos abandonan al organizador de los festejos a los que asistían asiduamente. En este relato, cuando Berenice se mira horrorizada al espejo, después de la desaparición de su hermosa cabellera, ve que la han dejado sola, que la han abandonado todos los miembros del grupo que hace unos momentos la aclamaban.

La educación sentimental que recibe Bernice durante la visita la convierte en lo que ya era su prima: la triunfadora del grupo. Al final de la historia las dos jóvenes son brillantes, egoístas, admiradas y pasarán a engrosar ese tipo femenino creado por Fiztgerald.

El aspecto estilístico más importante del relato es el tono irónico, que llega hasta lo trágico, $\mathrm{y}$ - como en su novela más conocida- demuestra que una sociedad de jóvenes adinerados y ociosos, que sólo piensan en divertirse, está muy lejos de representar «el sueño americano»: no se merecen la «nueva Jeru-

44 WAY, BRIAN. 1980 F. Scott Fitgerald and the ar of Social Fiction. op. cit., pag. 56. 
salén» ni el «Paraíso terrenal» americano, sino un mundo mezquino donde sólo tenga cabida la venganza.

\section{LOS «GRotescos» Y La Falta de COMUNICACióN}

Winesburg, Ohio muestra cómo transcurre la vida en un pueblo americano, con su capacidad para el sufrimiento, para las emociones, para la falta de comunicación: trata de todos los problemas comunes y subjetivos, que también pueden encontrarse en cualquier otra ciudad de América, aunque Anderson utilice el entorno material que le resultaba más familiar del Oeste Medio, donde se ha criado. En esta obra, según David Stouck, «Anderson achieves the greatest gains artistically through self-reflexive narration ${ }^{45}$ : George Willard, el reportero del periódico del pueblo, va entrando en relación con todos los personajes del libro, y éste será el hilo que conectará una con otra las historias hasta convertirlas en una novela cerrada. A través del contacto con los "grotescos» o personajes marginados de Winesburg, el joven escritor se hará adulto.

Al comienzo de la obra, Anderson explica que «when a person tries to possess a truth and live by it» se convierte en 'grotesco' $y$ «the truth embraced becomes a falsehood» ${ }^{46}$, pero, según Robert Dunne:

... upon reading the rest of Winesburg tales, we are left with the impression that Anderson incompletely spells out this condition, because while the symptoms are openly diagnosed by the old writer, a cure is not prescribed $^{47}$.

En $\ll H a n d s{ }^{48}$, la actitud del narrador hacia Wing es benévola y justifica sus defectos, presentándole como una figura pequeña y blanca que no sólo ins-

45 STouck, DAviD. 1985 «Sherwood Anderson and the Postmodem Novel», Contemporary Literature, XZ, XVI, 3, Fall, pág. 315.

46 ANDERSON, SHERWOOD. 1947 (1919) Winesburg, Ohio (A Group of Tales of Ohio SmallTown Life) US: The Modern Library, pág. 24.

47 DUNNE, RoberT. 1990 «Beyond Grotesqueness in Winesburg. Ohio», en The Midwest Quarterly-A Journal of Contemporary Thought. Pittisburg, Kansas: Pittsburg State University, Winter, Volume XXXI, Number 2, pág. 180.

48 ANDERSON, SHERWOOD. 1947 (1919) «Hands», Winesburg, Ohio, ed. cit. 
pira desprecio, sino también lástima, conmoviendo los corazones de sus vecinos: «In Winesburg the hands had attracted attention merely because of their activity... They became his distinctive feature, the source of his fame ${ }^{49}$. Incluso la latente homosexualidad del ambivalente maestro de escuela se explica como una gran vocación hacia los niños. Es un idealista, un soñador que anhela volver a vivir en una Edad de Oro pastoril, siendo su sueño recurrente la escena tan querida de «clear-limbed young men, some afoot, some mounted upon horses. In crowds the young men came to gather about the feet of an old man who sat beneath a tree in a tiny garden and who talked to them» 50 ; pero esta Arcadia no se parece en nada a Winesburg y sus habitantes.

No obstante, también se observa un tono crítico del narrador hacia el viejo maestro: a pesar de que la sociedad deba comprender esos defectos, él es culpable por no tratar de comunicarse con sus conciudadanos y permanecer escondido en casa. Sin embargo, el mensaje de la historia no es pesimista; puesto que todos los grotescos le han contado a George Willard sus sufrimientos, sus complejos, sus luchas, hay una promesa velada de posibilidad de salvación a través de un acto de amor: cuando el joven reportero abandona el pueblo y se convierte en el representante y portavoz de todos sus amigos que se quedan allí aislados, el escritor será la voz de esos hombres y mujeres que le han confiado sus secretos, y curarán sus deformidades a través de esta forma de comunicación.

\section{MANipulación del Lector En El TRATAMIENTo de la Violencia}

Las obras escritas después de la segunda Guerra Mundial muestran una tendencia, no sólo a evitar los finales felices, sino incluso a sorprender al lector con un giro completo en la dirección del argumento: «The lottery» ${ }^{51}$, de Shirley Jackson, y «A Good Man is Hard to Find $»^{52}$, de Flannery O'Connor, son historias divertidas al principio y continúan así hasta que, de pronto, se convierten en trágicas. Sin embargo, hay varios indicios que nos dan la clave para vislum-

49 Ibid. pág. 29.

50 Ibid, pág. 30.
51 JaCKSON, ShiRLEY. 1948 «The Lottery» The New Yorker, US: Farrar, Strauss \& Giroux, Inc.

52 O'Connor, Flannery. 1953 «A Good Man Is Hard to Find», A Good Man Is Hard to find and Other stories. US: Harcourt Brace Javanovich, Inc. 
brar que algo va a ocurrir en el momento álgido de la historia y, por tanto, el final no va a ser tan feliz como parecía al comienzo.

El relato de Jackson quizá sea una de las obras que han merecido mayor atención y su análisis ha despertado mayor interés, debido quizás a la forma en que están presentados los acontecimientos, que cautivan y sorprenden al lector. Según Gordon Weaver:

Few readers have failed to recognize the very real element of human depravity in Jackson's story-of the scapegoat on whom all the evils of the community are laid. In this respect, the story is a telling and chilling indictment of American society ${ }^{53}$.

La obra comienza con una pintura realista de la vida de un pueblo. Los vecinos se preparan para un acontecimiento que no se describe y que parece formar parte de las tradiciones del pueblo. Son personajes amistosos que se gastan bromas, mientras un narrador irónico anuncia que la «lottery» terminará pronto - a fin de que los aldeanos puedan volver a casa a la hora del almuerzo- siendo los chicos los primeros en llegar a la plaza «gathering a pile of stones».

Hay una confrontación Norte/Sur muy significativa para tratar de abolir la tradición, en la que el Norte siempre representa el progreso y el avance: en el Sur no hay cambios, es como el viejo Warner, que llama a los norteños «pack of crazy fools», protestando porque en vez de no olvidar el antiguo refrán: «Lottery in June, corn be heavy soon», tan sólo escuchan a los jóvenes. Este proverbio es la clave principal de la historia y aclara el motivo de la "lotería» anual: una celebración cruenta derivada directamente de tiempos ancestrales cuando se ofrecían sacrificios con ofrendas a los dioses- que ha pasado de generación en generación a través de los siglos, porque nadie se ha atrevido a cortar la tradición por miedo a las consecuencias. Todos los años los «Adams», o individuos progresistas, resultaban vencidos por los «Summers», que exigían a la comunidad no romper las tradiciones.

Todo esto parece bastante inocente hasta el clímax de la historia, cuando el realismo se convierte en simbolismo y la expectación se vuelve sobresalto al comprobar que la gente del pueblo mata a pedradas a uno de los vecinos, a

53 Weaver, Gordon (ed.) 1983 The American Short Story (1945-1980): A Critical History. Boston, Mass: Twayne Publishers, pág. 10. 
fin de saldar el pago del tributo humano. ¿Hasta cuándo va a continuar el pueblo esta tradición cruenta? La historia no resuelve este enigma y, por tanto, el lector saca la conclusión de que la sangrienta «lotería» seguirá celebrándose: estos sureños no son capaces de cambiar sus costumbres, aunque la pobre mujer elegida para chivo expiatorio de este sacrificio de violencia ritual, repita:

«It isn't fair, it isn't right. But although the villagers had forgotten the ritual and lost the original black box, they still remembered to use the stones $^{54}$, ,

En el relato de O'Connor, la tensión entre lo sacramental y lo grotesco es una de las caracterizaciones del lenguaje, y la técnica más utilizada es el simbolismo. Según Frederick Asals: «the tension between outer and inner, the seen and the unseen, is so radical in her work that a pervasive doubleness reaches into almost every cornern ${ }^{55}$.

Esta obra de O'Connor gira en torno a Misfit, un personaje complejo: un intruso; la oveja negra de la familia, que no recuerda el crimen que le llevó a la cárcel, esa solitaria y reducida celda donde ha permanecido durante años y donde no quiere volver: «turn to the right, it was a wall ... turn to the left, it was a wall. Look up it was a ceiling, look down it was a floon ${ }^{56}$.

La descripción que nos hace el narrador de la abuela es crítica, ya que como afirma Marshall Bruce Gentry:

The narrator consistently implies that the grandmother is responsible for the massacre of her family by the Misfit. But the narrator's misleading emphasis on the grandmother as the agent of the catastrophe ultimately makes apparent just how little the grandmother has to do with bringing about even her own awakening ${ }^{57}$.

54 JACKSON, SHIRLEY. 1948 «The Lottery», ed., cit., pág. 519.

55 ASALS, FREDERICK. 1982 Flannery O'Connor: The Imagination of Extremity. Athens, Georgia: The University of Georgia Press, pág. 72.

56 O'CONNOR, FlanNerY. 1953 «A Good Man Is Hard to Find», ed., cit., pág. 916.

57 GentRY, MarShall BRUCE. 1986 Flannery $O^{\prime}$ Conor's Religion of the Grotesque. Jackson \& London: University Press of Mississippi, pág. 21. 
Hay fuertes implicaciones religiosas. Misfit recuerda que «Jesus was the only One that ever raised the dead... and He shouldn't have done it. He thrown everything off balance» ${ }^{58}$. Está convencido de que, al igual que Jesús, también él ha sufrido sin merecerlo, aunque exista una diferencia entre ellos, ya que «He hadn't committed any crime» ${ }^{59}$.

La descripción que nos presenta el narrador de Misfit es benévola: a pesar de la crueldad del personaje, el narrador manipula al lector - resalta que llevaba «silver-rimmed spectacles that gave him a scholarly look»- para poder sorprenderle al final de la historia, donde la divertida comedia de la primera mitad de la obra se ensombrece hasta convertirse en una comedia negra, en la que perecen violentamente los seis miembros que componen la familia.

Estos finales sorprendentes contrastan con el desarrollo amable de las dos historias. Ambas son un ejemplo de habilidad de sus autores para sorprender al lector mientras llevan a sus máximas consecuencias el tema de la violencia: en la primera, a través del análisis de los sentimientos y emociones producidas por tradiciones y costumbres ancestrales, y en la segunda, tratando de hallar los responsables de que la sociedad produzca tipos tan inadaptados como Misfit.

Este breve recorrido por la novela corta americana pone de manifiesto la extraordinaria dificultad de este género, donde cada palabra tiene que ser exacta, justa y precisa, como claramente demuestra «The story of an Hour». Pone igualmente de manifiesto su preferencia por los temas claramente americanos: hay muchas preguntas sobre problemas morales y pocas respuestas o conclusiones - lo que nos acerca a la actitud puritana - en las limitaciones y deformaciones humanas de los personajes inadaptados de Flannery O'Connor o los grotescos de Sherwood Anderson.

Si la novela corta ha conseguido llegar a ser un gran género literario en Norteamérica es porque no se limita a narrar simples anécdotas de forma lineal; precisamente la dimensión que la caracteriza es que el escritor se coloca en el centro de la acción y nos la presenta iluminada, rodeada de misterio y vitalidad: puede servir de ejemplo la visión de Shirley Jackson de la sociedad dentro de un marco reducido y cerrado, pero magnificado por cada detalle, mientras van analizando los sentimientos y emociones de esas gentes sencillas del pueblo a través de una lupa minuciosa; o la tensión dramática y densidad que utiliza Willian Faulkner para acentuar la voz de la comunidad en «A Rose for Emily».

58 O'Connor, Flannery. 1953 *A Good Man is Hand to Find», ed., cit., pág. 916.

59 Ibid, pág. 916. 
No puede ponerse en duda la vitalidad de la novela corta norteamericana, que sigue investigando y descifrando, comentando e interpretando, incluso anticipando la historia de su país. De ahí que Gordon Weaver llegue incluso a afirmar:

Contemporary American short stories are an idex of national consciousness: they focus on what it means to be human, and thus becomes an agent of perception with the capacity for influencing moral action in America today ${ }^{60}$.

Por tanto, no hay que preocuparse por la continuidad de la novela corta norteamericana. Su futuro está asegurado por el extraordinario plantel de autores contemporáneos que eligen este género literario como forma de expresión artística, y que sigue produciendo esa literatura fresca y original, rica en leyendas y mitos.

60 Weaver, Gordon. ob. cit., pág. 6. 